\title{
ANÁLISE DA ASSIMETRIA FUNCIONAL HEMISFÉRICA DE CRIANÇAS COM EPILEPSIA PARCIAL E QI NORMAL PELO DESEMPENHO NOS SUBTESTES DO WISC
}

MAURO MUSZKAT * - NEYDE SANTACCHI DE VINCENZO * - ALICE MASUKO *

DENILCE O. REAMI * - CARLOS J. REIS DE CAMPOS *

\begin{abstract}
RESUMO - Foram avaliadas 26 crianças, sem deficiência mental, com média de idade de $9 \pm 2$ anos, com epilepsia parcial sem lesão cerebral evidenciável à TCC e com atividade piaroxistica no EEG intercrítico localizada apenas em um hemisfério cerebral. Essas crianças foram submetidas à Escala de Inteligência Wechsler para Crianças (WISC) e à análise dos vários subtestes verbais e execução em relação à localização hemisférica da descarga no EEG. Os dados foram comparados ao desempenho no WISC de um grupo de 61 crianças sem epilepsia na mesma faixa etária (Grupo Controle). As crianças epilépticas apresentaram desempenho inferior no QI execução, em três subtestes verbais (Informação, Dígitos e Aritmética) e em três subtestes execução (Cubos, Armar Objetos e Código). Os pacientes com foco eletrencefalográfico no hemisfério direito apresentaram desempenho inferior ao Grupo Controle nos subtestes relacionados à habilidade espacial e atenção não-verbal (Cubos, Armar Objetos e Código), enquanto as crianças com foco no hemisfério esquerdo apresentaram desempenho inferior apenas no subteste de Dígitos (relacionado à memória imediata e atenção verbal). Os autores sugerem que a localização hemisférica do foco no EEG intercrítico tem relação com o tipo de déficit cognitivo associado à epilepsia parcial não-lesional, mesmo em crianças com QI normal.
\end{abstract}

Functional hemispherical asymmetry with WISC analyses in children with partial epilepsy and normal IQ

SUMMARY - We studied 87 normal IQ children, 26 partial epileptic (epileptic group) and 61 non-epileptic children (control group) with mean age of 9 years in relation to cognitive performance in Wechsler Infant Scale for Children (WISC) battery. The epileptic group showed lower score in IQ performance and in three verbal subtests (Information, Digit span, Arithmetic) and three performance subtests (Block Design, Object Assembly and Coding). Patients with right hemisphere EEG focus showed low score in subtests related to spatial ability and non-verbal attention, whereas patients with left hemisphere EEG focus showed low score only in Digit span subtest (related to immediate memory and verbal attention). The authors suggest that cognitive deficits in partial non-lesional epileptic children is related to the side of EEG interictal abnormalities, even in children with normal IQ.

O estudo dos vários aspectos do desempenho cognitivo de crianças epilépticas vem, nos últimos anos, assumindo espaço crescente nas investigações neuropsicológicas 3,4. A avaliação do perfil cognitivo de crianças com epilepsia fornece informações valiosas, de importância diagnostica, na avaliação das influências das crises epilépticas ou das várias drogas anti-epilépticas no desempenho cognitivo ${ }^{24}$, no prognóstico, na delimitação das epilepsias sintomáticas 10 e na terapêutica através da escolha de técnicas psicológicas, psicomotoras ou educacionais a serem implementadas para cada casoi*. As primeiras observações sobre a relação entre inteligência e epilepsia foram baseadas somente em impressões clínicas, muitas delas influenciadas pelas idéias dos alienistas do século XIX, que associavam a epilepsia à deficiência e deterioração mental !3. Com o desenvolvimento de testes neuropsicológicos e de inte-

* Setor de Investigação e Tratamento das Epilepsias, Disciplina de Neurologia, Escola Paulista de Medicina.

Dr. Carlos José Reis de Campos - Disciplina de Neurologia, Escola Paulista de Medicina Rua Botucatu 740 - 04023 São Paulo SP - Brasil. 
ligência, tal perspectiva comecou a modificar-se demonstrando que epilepsia não implica, necessariamente, em déficit intelectual ou psicológico e que muitas crianças epilépticas apresentam desempenho neuropsicológico global semelhante às crianças não epilépticas 2.9. A Escala de Wechsler para avaliação da inteligência de crianças, WISC, tem sido uma das medidas amplamente utilizadas. Embora, tradicionalmente, tenha sido utilizado para o levantamento quantitativo do nível intelectual, pode-se conseguir avaliar, pela análise qualitativa dos seus subtestes, o desempenho neuropsicológico dos indivíduos 7,20 . Além disso, permite analisar a assimetria funcional hemisférica por um levantamento qualitativo de alguns subtestes verbais ligados mais intimamente ao hemisfério esquerdo (Semelhanças, Dígitos) e de alguns subtestes execução, relacionados às funções espaciais do hemisfério direito (Cubos, Armar Objetos) 7,20 .

O objetivo do presente estudo é avaliar o desempenho neuropsicológico considerando as diferenças relativas à assimetria funcional hemisférica, de crianças com epilepsia parcial, em relação a grupo controle não-epiléptico, a partir da escala global de inteligência e da análise do desempenho qualitativo nos vários subtestes do WISC?

\section{CASUISTICA E METODOLOGIA}

O Grupo Estudo foi constituído de 26 pacientes $(6$ a 16 anos $)$, com média de idade de $9 \pm 2$ anos, 21 do sexo masculino e 5 do sexo feminino, frequentando escola pública (1» a 8» série do $\boldsymbol{l} \boldsymbol{v}$ grau), com epilepsia parcial classificada de acordo com os critérios internacionais da 1 LAE 6, sem lesão cerebral evidenciável ao exame de tomografia computadorizada do crânio (TCC). O Grupo Controle foi selecionado a partir de 120 questionários dirigidos aos pais de crianças de uma escola pública estadual, na mesma faixa de escolaridade do Grupo Estudo. A partir dos questionários, foram selecionadas 61 crianças, sem suspeita de deficiência mental e sem suspeita de distúrbio neurológico, na faixa etária de 7 a 15 anos, com média de idade de $9 \pm 2$ anos.

Todos os casos do Grupo Estudo apresentavam atividade paroxística no Eeg inter crítico localizada em apenas um hemisfério cerebral, sem difusão para o hemisfério contralateral. Após avaliação clínica e neurológica, todos os casos do Grupo Estudo e do Grupo Controle realizariam exame neuropsicológico, que incluia avaliação do QI pela Escala de Inteligência de Wechsler para crianças (WISC) e pela análise qualitativa do desempenho nos subtestes verbais e execução da mesma escala 7.

Foram considerados apenas os pacientes com QI (Quociente de Inteligência) > 70 . Os dados obtidos foram analisados em relação às diferenças de desempenho cognitivo do Grupo Estudo e do Grupo Controle, bem como em relação à influência da localização da atividade paroxística no EEG intercrítico sobre o desempenho neuropsicológico. A análise do WISC incluiu - (a) QI total, QI verbal, QI execução, Discrepância verbal-execução; (b) Desempenho nos 10 subtestes: 5 verbais (Informação, Compreensão, Semelhanças, Aritmética, Dígitos) e 5 execução (Completar Figuras, Armar Objetos, Cubos, Arranjo de Figuras e Código).

\section{RES ULT A D OS}

Na Tabela 1 são mostradas algumas características do Grupo Estudo. Observa-se nítido predomínio da epilepsia parcial idiopática $(18 / 26 ; 69,2 \%$ dos casos). Todos os pacientes do Grupo Estudo faziam uso de drogas antiepilépticas: 23 casos $(23 / 26 ; 88,5 \%)$ estavam em monoterapia, em Sua maioria $(19 / 26 ; 73,1 \%)$ com fenobarbital (Tabela 1).

Na Tabela 2 são mostrados os resultados obtidos no WISC nos Grupos Controle e Estudo. Observa-se que o Grupo Estudo não apresentou no QI total diferenças significantes em relação ao Grupo Controle. O desempenho do QI execução, nos casos do Grupo Estudo, foi inferior ao Grupo Controle. Em relação ao desempenho nos subtestes observa-se que o Grupo Estudo apresetntou desempenho inferior ao Grupo Controle no subteste verbal de Dígitos e no subteste execução de Completar Figuras.

Analisando a lateralidade da descarga no EEG intercrítico com o desempenho cognitivo no WISC (Tabela 3), observa-se que os casos com atividade paroxística no hemisfério direito apresentaram desempenho inferior no QI execução, tanto em relação aos casos com atividade paroxística temporal esquerda, quanto ao Grupo Controle não-epiléptico. Em relação ao desempenho nos subtestes verbais e execução do WISC, observamos que os casos com pa- 


\begin{tabular}{|c|c|c|}
\hline Tipo de Crise & $\mathbf{N}$ & $\begin{array}{l}\text { Frequên- } \\
\text { cia \% }\end{array}$ \\
\hline PS & $\mathbf{5}$ & 19,3 \\
\hline $\mathbf{P C}$ & 1 & $\mathbf{3 , 7}$ \\
\hline $\mathbf{P} \rightarrow \mathbf{G}$ & 10 & 38,5 \\
\hline$P S+P \rightarrow G$ & 10 & 38,5 \\
\hline \multicolumn{3}{|l|}{ Tipo de Epilepsia } \\
\hline Idiopática & 18 & 69,2 \\
\hline Criptogenética & 8 & 30,8 \\
\hline \multicolumn{3}{|l|}{ Lateralização EAEG } \\
\hline Direita & 12 & 46,2 \\
\hline Esquerda & 14 & 53,8 \\
\hline \multicolumn{3}{|l|}{ Tratamento } \\
\hline Fenobarbital & 19 & 73,1 \\
\hline Carbamazepina & 3 & 11,5 \\
\hline Valproato & 1 & 3,9 \\
\hline Politerapia & 3 & 11,5 \\
\hline Total & 26 & 100 \\
\hline
\end{tabular}

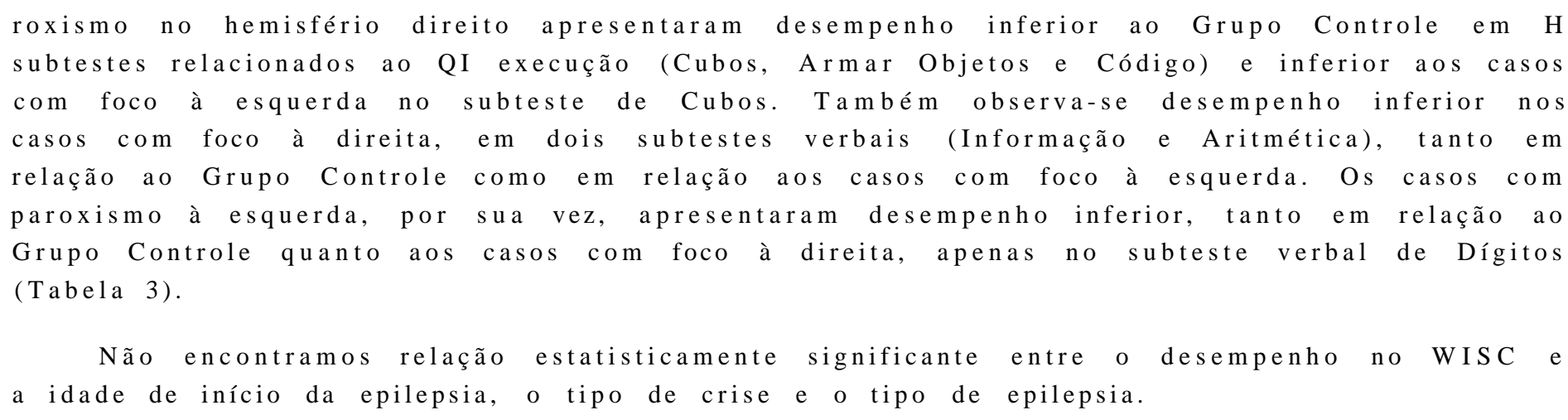

\section{COMENTÁRIOS}

Neste estudo, não observamos diferenças entre o desempenho cognitivo global do Grupo Controle e do Grupo Estudo, traduzido no Quociente de Inteligência (QI) Total. No entanto, a partir da análise dos subtestes verbais e de execução, observamos que o Grupo Estudo apresentou desempenho inferior ao Grupo Controle no Ql execução, em um subteste da escala verbal (Dígitos) e em um subteste da escala execução (Completar Figuras) (Tabela 2).

Farwell e Dodrilis, estudaram o desempenho cognitivo, através do WISC, em um grupo de crianças com $Q I>70 ; 118$ crianças eram epilépticas e as de um grupo controle não apresentavam epilepsia, como neste estudo. Observaram que as crianças epilépticas, incluindo 31 com epilepsia parcial, apresentavam QI total inferior às não epilépticas. Posteriormente, Ellenberg et al.\&, comparando o desempenho cognitivo através do WISC em grupo heterogêneo de 98 crianças epilépticas (QI>70), em relação a seus irmãos não-epilépticos, não encontraram diferenças no QI Total, sugerindo que as crises epilépticas não interferem na capacidade intelectual global. Esses estudos apresentam, no entanto, diferenças de natureza metodológica, pois avaliam grupo heterogêneo de casos em relação ao tipo de crise e tipo de epilepsia. Na série de Farwell e Dodrill, no grupo com epilepsia parcial houve predomínio de casos de 


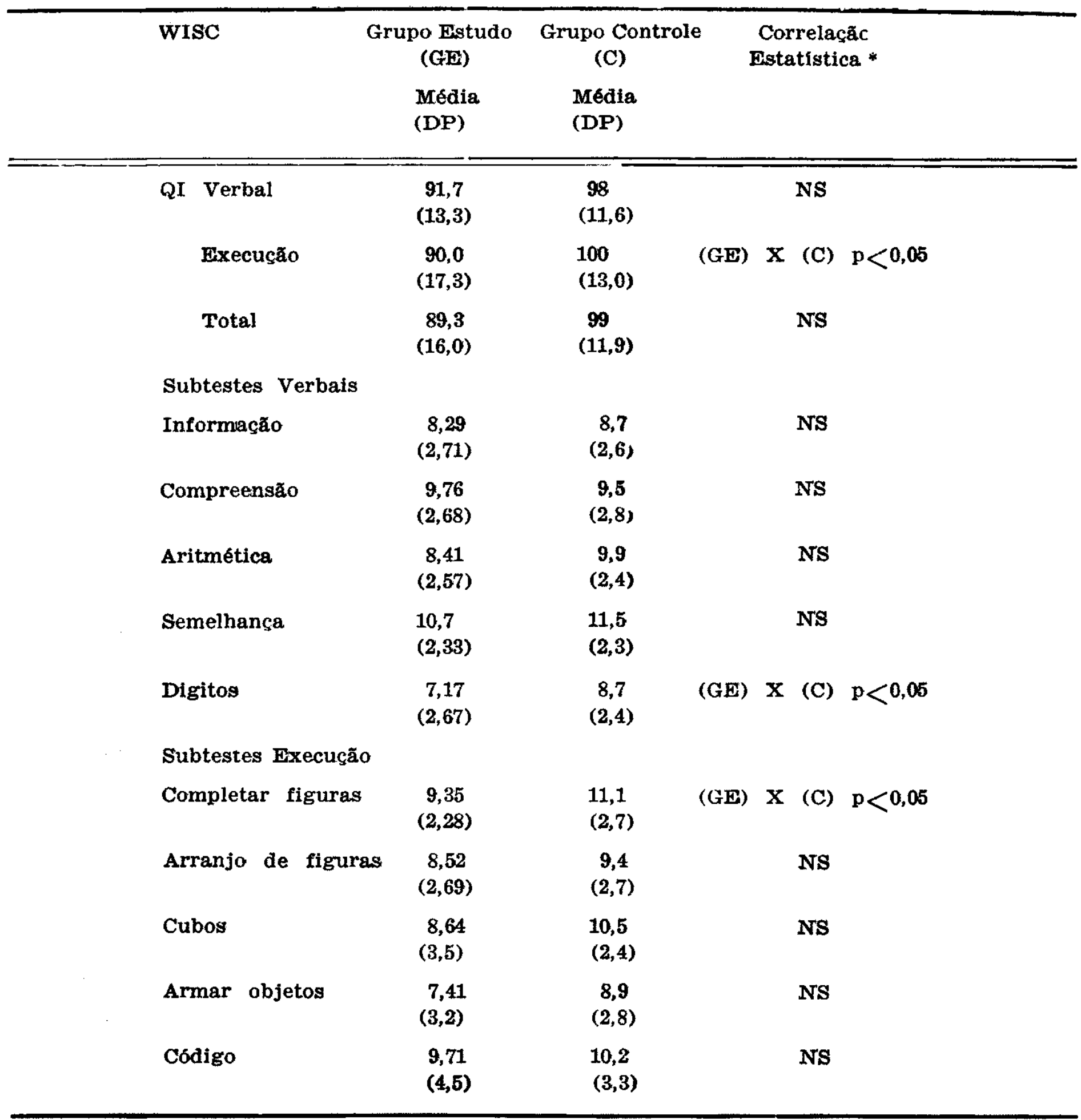

Tabela 2 - Andise qualitativa do WISC (Grupo Estudo $\times$ Grupo Controle).

* Teste de Mann-Whitney, teste de Kruskal-Wallis e teste de Comparagres Multiplas. NS, não significativo; $D P$, desvio padrāo.

epilepsia parcial sintomática com crises parciais complexas, enquanto na série de Ellenberg et al., como também em nossa série, houve predomínio de casos de epilepsia parcial idiopática (Tabela 1). Dado que as crianças com epilepsia não formam grupo homogêneo, os déficits cognitivos observados devem sempre ser analisados dentro de contexto multifatorial, que inclua os vários tipos de epilepsia 28 a presença de lesão cerebral associada ${ }^{2}$, o tipo de droga anti-epiléptica empregada^

$O$ fato de não termos encontrado relação entre os déficits cogniticos e idade de início, tipo de crise e tipo de epilepsia se deve, provavelmente, às características dos casos estudados, pois analisamos grupo bastante homogêneo de crianças, principalmente em idade escolar, com epilepsia parcial não lesionai em monoterapia com fenobarbital (Tabela 1).

Observamos nesta investigação que as crianças com epilepsia parcial, mesmo na ausência de lesão cerebral, apresentam maior frequência de déficit cognitivo específico, principalmente relacionado à organização perceptiva dos estímulos espaciais e à retenção dos estímulos sensoriais ligados à atenção e memória, o que também foi observado por outros autores 26-28. 


\begin{tabular}{|c|c|c|c|c|c|c|c|}
\hline \multirow[t]{2}{*}{ WISC } & \multicolumn{2}{|c|}{$\begin{array}{c}\text { Grupo Estudo } \\
\text { Foco EEG }\end{array}$} & \multirow{2}{*}{\multicolumn{2}{|c|}{ Grupo Controle }} & \multirow{2}{*}{\multicolumn{3}{|c|}{$\begin{array}{l}\text { Correlação } \\
\text { Estatística }\end{array}$}} \\
\hline & $\begin{array}{l}\text { Dirgito (D) } \\
\text { Média } \\
\text { (DP) }\end{array}$ & $\begin{array}{c}\text { Esquerdo (E) } \\
\text { Média } \\
\text { (DP) }\end{array}$ & & & & & \\
\hline QI Verbal & $\begin{array}{l}86,2 \\
(9,6)\end{array}$ & $\begin{array}{c}94,5 \\
(14,9)\end{array}$ & $\begin{array}{c}98 \\
(11,6)\end{array}$ & & & NS & \\
\hline Execução & $\begin{array}{c}84,2 \\
(14,5)\end{array}$ & $\begin{array}{c}90,6 \\
(16,2)\end{array}$ & $\begin{array}{l}100 \\
(13,0)\end{array}$ & $\begin{array}{l}\text { (D) } \\
\text { (D) }\end{array}$ & $\begin{array}{l}\mathbf{x} \\
\mathbf{x}\end{array}$ & $\begin{array}{l}\text { (C) } \\
\text { (E) }\end{array}$ & $\begin{array}{l}p<0,01 \\
p<0,01\end{array}$ \\
\hline Total & $\begin{array}{c}83,5 \\
(11,1)\end{array}$ & $\begin{array}{c}91,6 \\
(16,5)\end{array}$ & $\begin{array}{l}99 \\
(11,9)\end{array}$ & & & NS & \\
\hline \multicolumn{8}{|l|}{ Subtestes Verbais } \\
\hline Informação & $\begin{array}{c}6,4 \\
(2,3)\end{array}$ & $\begin{array}{c}8,0 \\
(3,2)\end{array}$ & $\begin{array}{c}8,7 \\
(2,6)\end{array}$ & $\begin{array}{l}\text { (D) } \\
\text { (D) }\end{array}$ & $\begin{array}{l}\mathbf{X} \\
\mathbf{X}\end{array}$ & $\begin{array}{l}\text { (C) } \\
\text { (E) }\end{array}$ & $\begin{array}{l}p<0,05 \\
p<0,05\end{array}$ \\
\hline Compreensão & $\begin{array}{c}8,0 \\
(2,0)\end{array}$ & $\begin{array}{l}10,2 \\
(2,9)\end{array}$ & $\begin{array}{c}9,5 \\
(2,8)\end{array}$ & & & NS & \\
\hline Aritmética & $\begin{array}{c}7,1 \\
(2,2)\end{array}$ & $\begin{array}{c}8,8 \\
(3,2)\end{array}$ & $\begin{array}{c}9,9 \\
(2,4)\end{array}$ & $\begin{array}{l}\text { (D) } \\
\text { (D) }\end{array}$ & $\begin{array}{l}\mathbf{x} \\
\mathbf{x}\end{array}$ & $\begin{array}{l}\text { (C) } \\
\text { (E) }\end{array}$ & $\begin{array}{l}p<0,01 \\
p<0,05\end{array}$ \\
\hline Semelhança & $\begin{array}{c}9,9 \\
(2,1)\end{array}$ & $\begin{array}{l}11,3 \\
(2,0)\end{array}$ & $\begin{array}{l}11,5 \\
(2,3)\end{array}$ & & & NS & \\
\hline Digitos & $\begin{array}{c}7,5 \\
(1,7)\end{array}$ & $\begin{array}{c}6,5 \\
(3,3)\end{array}$ & $\begin{array}{c}8,7 \\
(2,4)\end{array}$ & $\begin{array}{l}\text { (E) } \\
\text { (D) }\end{array}$ & $\begin{array}{l}\mathbf{x} \\
\mathbf{x}\end{array}$ & $\begin{array}{l}\text { (C) } \\
\text { (E) }\end{array}$ & $\begin{array}{l}p<0,01 \\
p<0,05\end{array}$ \\
\hline \multicolumn{8}{|l|}{ Subtestes Execução } \\
\hline Completar figuras & $\begin{array}{c}9,7 \\
(3,0)\end{array}$ & $\begin{array}{c}9,8 \\
(2,2)\end{array}$ & $\begin{array}{l}11,1 \\
(2,7)\end{array}$ & & & NS & \\
\hline Arranjos de figuras & $\begin{array}{c}7,0 \\
(2,9)\end{array}$ & $\begin{array}{c}8,5 \\
(2,1)\end{array}$ & $\begin{array}{c}9,4 \\
(2,7)\end{array}$ & & & NS & \\
\hline Cubos & $\begin{array}{c}7,5 \\
(1,6)\end{array}$ & $\begin{array}{c}9,5 \\
(3,1)\end{array}$ & $\begin{array}{l}10,5 \\
(2,4)\end{array}$ & $\begin{array}{l}\text { (D) } \\
\text { (D) }\end{array}$ & $\begin{array}{l}\mathbf{x} \\
\mathbf{x}\end{array}$ & $\begin{array}{l}\text { (C) } \\
\text { (E) }\end{array}$ & $\begin{array}{l}p<0,01 \\
p<0,06\end{array}$ \\
\hline Armar objetos & $\begin{array}{r}7,0 \\
(2,3)\end{array}$ & $\begin{array}{c}7,5 \\
(2,5)\end{array}$ & $\begin{array}{c}8,9 \\
(2,8)\end{array}$ & (D) & $\mathbf{x}$ & (C) & $\mathrm{p}<0,01$ \\
\hline Código & $\begin{array}{c}7,5 \\
(4,1)\end{array}$ & $\begin{array}{c}8,0 \\
(4,0)\end{array}$ & $\begin{array}{c}10,2 \\
(3,3)\end{array}$ & (D) & $\mathbf{x}$ & (C) & $p<0,01$ \\
\hline
\end{tabular}

Tabela 3 - Andise qualitativa do WISC e lateralidade do foco.

* Teste de Mann-Whitney, teste de Kruskal-Wallis e teste de Comparaçes Múltiplas. NB, nlo significativo; DP, desvio padrao.

Em nosso estudo, o pior desempenho no QI execução, comparado ao QI verbal encontrado no Grupo epiléptico, pode relacionar-se a dificuldades de percepção visual e organização espacial ou à menor velocidade do desempenho motor (Armar Objetos, Cubos e Código). É importante ressaltar que, na contagem do QI execução, um dos principais parâmetros é o tempo de realização das tarefas. Portanto, o QI execução pode ter sido influenciado pelos efeitos da medicação anti-epiléptica sobre a velocidade das respostas motoras em provas de discriminação de estímulos sensoriais. Alguns autores relatam que, dentre as drogas anti-epilépticas, é principalmente o fenobarbital que pode ter efeitos sobre a coordenação visuomotora 26. No entanto, Smith e Graft28 acreditam que as drogas não podem ser inteiramente responsabilizadas pois, em sua casuística, mesmo os indivíduos epilépticos não tratados apresentaram desempenho motor inferior aos do grupo controle não-epiléptico.

Ao realizarmos uma análise qualitativa do desempenho nos subtestes do WISC, observamos que as dificuldades apresentadas pelo Grupo Estudo relacionaram-se, prin- 
cipalmente, a fatores que envolvem atenção-concentração (Aritmética, Dígitos e Código), memória imediata (Dígitos), percepção visual, análise e síntese, organização espacial e ordenação das respostas visuomotoras (Armar Objetos, Cubos e Código).

O subteste Informação relaciona-se à memória remota e à fluência verbal, mas é um dos subtestes mais sujeito a fatores culturais e ambientais, o que o torna pouco específico em termos de análise neuropsicológica. O subteste de Dígitos é uma medida confiável de memória verbal imediata: envolve atenção, flexibilidade mental na repetição da ordem inversa e capacidade de sequenciação 12. Segundo Kaufman \& Reynolds 18, os subtestes de Aritmética, Código e Dígitos estão intimamente relacionados entre si, pois os três subtestes são sensíveis às alterações da capacidade de atenção-concentração, no que se refere a resistência à distraibilidade. Os subtestes de Cubos e Armar Objetos relacionam-se à percepção visual, organização espacial e ordenação visuomotora, pois envolvem análise e síntese visual na reprodução e orientação de figuras espaciais simples e complexas; além disso, associam-se mais ao desempenho do hemisfério direito nos indivíduos destros 18. o subteste de Código relaciona-se,-principalmente, à capacidade de atenção visual e destreza visuomotora, envolvendo um dos aspectos principais da atenção: a seletividade 30 .

Vários fatores podem influenciar a capacidade de atenção e memória nas crianças com epilepsia parcial i?. Tais fatores incluem a presença de lesão cerebral associada, o uso de drogas anti-epilépticas, crises repetidas levando a alterações neurotóxicas pelo acúmulo de excitoxinas no foco epiléptico e alterações neuropatológicas como perda neuronal no hipocampo'?. Glowinskii ${ }^{3}$ considera que, mesmo descargas paroxísticas subictais na região temporal, podem prejudicar a memória por influenciar ou interromper a capacidade de armazenamento de informações.

Alguns estudos i» 5 têm sugerido influências da lateralização da atividade paroxística no EEG sobre o tipo de déficit cognitivo observado, com diminuição do desempenho verbal nos focos à esquerda e do desempenho espacial nos focos à direita. Em nosso estudo, também encontramos essa relação entre a lateralização da descarga paroxística e o tipo de déficit cognitivo observado. Os casos com atividade paroxística localizada no hemisfério direito apresentaram desempenho inferior nas provas relacionadas à percepção e organização espacial, ordenação visuomotora e atenção, funções neuropsicológicas habitualmente atribuídas ao hemisfério não dominante 16. Por outro lado. os casos com atividade paroxística no hemisfério esquerdo apresentaram desempenho inferior apenas no teste de Dígitos, que está intimamente relacionado à memória verbal e. portanto, ao hemisfério dominante para a linguagem 16.1».

Na maioria dos estudos que avaliam o desempenho cognitivo no período intercrítico de crises parciais são encontradas influências inibitórias das descargas localizadas sobre $o$ desempenho $\operatorname{cognitivo~}^{8}>\mathbf{i}^{7}$. $O$ déficit cognitivo transitório durante as descargas intercríticas previamente descrito em epilepsias generalizadas com ponta-onda $3 \mathrm{~Hz}^{1}$, tem sido também observado em epilepsias parciais 1.3. Os pacientes epilépticos da série de Aarts et ai. ${ }^{1}$ foram submetidos a monitorização eletrencefalográfica durante a avaliação do desempenho em «videogames», que incluíam provas de memória imediata utilizando material verbal e espacial. Nesse estudo ${ }^{1}$ foi documentada a presença de alterações cognitivas intercríticas durante descargas focais em 50\% dos pacientes estudados. Também foi encontrada associação entre a natureza da prova e a lateralização das descargas no EEG. As descargas no hemisfério direito causavam déficit nas provas espaciais, enquanto as descargas no hemisfério esquerdo estavam associadas a menor desempenho nas provas verbais.

Finalizando, consideramos que, apesar do WISC não ter sido desenvolvido especificamente para análise da assimetria funcional hemisférica, ele se mostrou útil na avaliação neuropsicológica de crianças com epilepsia parcial, pois não apenas mostrou o desempenho verbal e espacial global mas, também, permitiu a avaliação qualitativa das várias capacidades específicas. A análise dos efeitos das descargas epilépticas lateralizadas na assimetria funcional hemisférica pode, por sua vez, auxiliar a escolha e orientação de medidas de reabilitação adequada para cada caso.

REFERENCES

1. Aarts HP, Binnie CD, Smith AM, Wilkings AJ. Selective cognitive impairment during focal and generalized epileptiform EEG activity. Brain, 1084, 107:293-308.

2. Bagley C. The educational performance of children with epilepsy. Br J Educat Psichol $1970,40: 82-3$. 
3. Bennet JL», Stores G. The mature of cognitive dysfunction in school children with epilepsy Acta Neurol Scand 1984, 69:79-82.

4. Black FW. Patterns of cognitive impairment in children with suspected and documented neurological disfunction. Percep Motor Skills 1974, 39:115-120.

5. Camfield PR, Gates R, Roñen «G, Camfiel C, Ferguson A, MacDonald GW. Comparison of cognitive ability, personality profile, and school success in epileptic children with pure right versus temporal lobe EEG foci. Ann Neurol 1984, 15:122-126.

6. Commission on Classification «and Terminology of the International League Against Epilepsy. Proposal for classification of epilepsies and epileptic syndromes. Epilepsia 1989, 30:389-399.

7. Cunha JA, Freitas NK, Raimundo MGB. Escalas Wechsler: mane.io clínico. In Cunha JA, Freitas NK, Raimundo MGB: Psicodiagnóstico. Porto Alegre: Artes Médicas 1986, 180-235.

8. Dodrill CB, Wilkus RJ. Neuropsychological correlates of the electroencephalogram in epileptics: generalized non-epileptiform abnormalities. Epilepsia 1987, 19:453-462.

9. Ellenberg JH, Hirtz DG, Nelson KB. Age at onset of seizures in young children. Ann Neurol 1984, 15:129-134.

10. Ellenberg JH, Hirtz DG, Nelson KB. Do seizures in children cause intelectual deterioration? N Engl J Med 1986, 314:1085-1088.

11. Farwell JK, Dodrill CB, Batzel LW. Neuropsychological abilities of children with epilepsy. Epilepsia 1985, 26:395-400.

12. Giordini B, Berent S, Sackellares JC, Rourke D, Seidenberg M, O'Leary DS, Dreifus FE, Bool TJ. Intelligence test performance of patients with partial and generalized seizures. Epilepsia 1985, 26:37-42.

13. Glowinski H. Cognitive deficits in temporal lobe epilepsy: an investigation of memory functioning. J Nerv Ment Dis 1973, 157:129-137.

14. Guerrant J, Anderson WW, Fischer A, Weinstein MR, Jaros RM, Deskins A. Personality in Epilepsy. Springfield: Thomas, 1962.

15. Hackney A, Taylor DC. A teachers questionnaire description of epileptic children. Epilepsia 1976, 17:275-278.

16. Heilman KM, Bowers D, Valenstein E, Watson RT. The right hemisphere neuropsychological functions. J Neurosurg 1986, 64:693-704.

17. Hermann BP. Neuropsychological functioning and psychopathology in children with epi lepsy. Epilepsia 1982, 23:545-554.

18. Kaufman AS, Reynolds CR. Clinical evaluation of intellectual function. In Weiner IB (ed): Clinical Methods in Psychology. New York: John Wiley \& Sons, 1963, p 100-151.

19. Kimura D. Cerebral dominance for speech in the nervous system. In Eeagles ELi (ed) Human Communication and its Disorders. New York: Raven Press 1975, Vol 3, p 365-371.

20. Lezak MD. Neuropsychological assesment. In Vinken PJ, Bruyn GW, Klawans HL (eds): Handbook of Clinical Neurology. New York: Elsevier 1985, Vol 45, p 515-530.

21. Loiseau P, Strube E, Signoret JL*. Memory and epilepsy. In Trimble ML, Reynolds EH (eds): Epilepsy, Behavior and Cognitive Function. New York: John Wiley \& Sons 1987, p $165-176$

22. Mazzucchi A, Vistintini D, Magnani G, Cattelani R, Parma M. Hemispheric prevalence in partial epileptic patients on perceptual and attentional task. Epilepsia 1975, 264:370-390

23. Milner B, Taylor L, Sperry R. Lateralized suppression of dichotically present digits after commisural section in man. Science 1968« 161:184-186.

24. Muszkat M. Estimulação Dicótica Consoante-Vogal (ED-CV) em Pacientes com Epilepsia Parcial. Dissertação de Mestrado, Disciplina de Neurologia Clínica, Escola Paulista de Medicina São Paulo, 1989

25. Reynolds EH. Mental effects of antiepileptic medication: a review. Epilepsia 1983, 24:84-95

26. Rodin EA, Schmaltz S. Twitty G. Intellectual function in patients with childhood epilepsy. Dev Med Child Neurol 1986, 28:25-33.

27. Sillanpaa M. Medical-social prognosis of children with epilepsy: epidemiological study analysis of 245 patients. Acta Paed Scand 1973, 237:3-104.

28. Smith DB, Graft RB. V Cooperative Study Group. Differential neurotoxicity of four major anticonvulsants. Ann Neurol 1985, 18:119.

29. Sperry RM. Some effects of disconnecting the cerebral hemispheres. Science 1982, 27 1223-1226.

30. Zimmerman IL. Interpretación clínica da la escala de inteligencia de Wechsler para adultos. Madrid, 1976 